\title{
LA RELATION ENTRE LE DROIT INTERNATIONAL PUBLIC ET LE DROIT PÉTROLIER : ÉTUDE DE L'AFFAIRE ENTRE LE TIMOR-LESTE ET L'AUSTRALIE
}

\section{ARTICLE ORIGINAL}

PEREIRA, Jeremias ${ }^{1}$

PITHAN, Lívia Haygert ${ }^{2}$

PEREIRA, Jeremias. PITHAN, Lívia Haygert. La relation entre le droit international public et le droit pétrolier : Étude de l'affaire entre le Timor-Leste et l'Australie. Revue scientifique pluridisciplinaire de la base de connaissances. 04 année, Ed. 12, vol. 02, pp. 31-51. décembre 2019. ISSN: 2448-0959, Lien d'accès: https://www.nucleodoconhecimento.com.br/droit/droit-public-international

\section{RÉSUMÉ}

Cet article vise à analyser le droit de la mer et du pétrole pour comprendre les raisons qui ont généré, pendant plus d'une décennie, le conflit entre le Timor-Leste et l'Australie concernant la définition des frontières maritimes définitives. Le Timor-Leste a déjà été exploité et envahi par plusieurs nations. En raison de l'abondance du pétrole et du gaz naturel, elle a souffert de son indépendance en 2002, et a lutté pour profiter de son territoire maritime jusqu'en 2018 , dans ce nouveau contexte du traité frontalier maritime signé entre le Timor-Leste et le Australie. La ligne d'équidistance médiane était le paramètre utilisé pour définir les frontières timoles définitives, accordant le droit de jouir de leur territoire maritime. Le pacte définitif sur les frontières a grandement contribué au développement du Timor-Leste, en raison de l'exploitation des richesses marines, en plus de reconnaître la nécessité pour le pays d'aider les compagnies

${ }^{1}$ Faculté de droit académique, Faculté de droit, Université Pontificale Catholique de Rio Grande do Sul - PUC-RS.

${ }^{2}$ Conseiller d'orientation. Doctorat en droit privé. Maîtrise en droit. Diplôme de droit. 
pétrolières à contribuer à l'évolution du Timor-Leste d'une manière spécifique et ponctuelle. Cela doit maintenant être ratifié par les parlements nationaux des deux pays. Le processus de ratification devrait être achevé en 2019. Le Timor-Leste cherche actuellement à négocier ses frontières maritimes avec l'Indonésie, mais celles-ci ont été suspendues pendant le processus de conciliation obligatoire avec l'Australie. Maintenant que ce processus est terminé, les deux pays peuvent reprendre leurs discussions.

Mots-clés: Droit pétrolier, droit international, droit maritime.

\section{INTRODUCTION}

Depuis plus d'une décennie, l'Australie et le Timor-Leste sont en désaccord avec les frontières maritimes de chacun de ces États. Le différend a lieu en raison du droit à l'exploration pétrolière et à d'autres richesses marines et des obstacles au développement économique et politique du Timor-Leste, après un traité signé avec l'Australie. Ainsi, cet article se développe dans le domaine du droit international public, en particulier dans le domaine du droit pétrolier.

On peut se demander si le traité entre l'Australie et le Timor-Leste, sur la définition des frontières maritimes, a été équilibré, préservant pleinement le droit des timorais. Quels sont les obstacles pour empêcher cette nation d'être exploitée de manière disproportionnée par d'autres nations et peut se développer à partir de 2019 ?

La pertinence de cette recherche est justifiée par le fait que la nécessité de respecter les frontières maritimes de timor-leste par l'Australie est justifiée. Au XXle siècle, malgré l'existence du droit international et du droit pétrolier, l'Australie n'avait aucun intérêt à mettre en œuvre un traité dans lequel les droits de timor-leste étaient reconnus pour recevoir le pourcentage approprié pour l'exploration pétrolière de territoire maritime. La fragilité économique historique du pays avec un espace terrestre beaucoup plus faible que celui des gens de mer en a fait la cible de l'exploitation de pays comme l'Indonésie et l'Australie. Bien qu'il y ait eu un traité permanent entre le Timor-Leste et l'Australie en 2018, il est nécessaire d'explorer plusieurs documents 
juridiques traitant du droit de la mer et du pétrole pour construire des bases juridiques internationales, dans le but de se multiplier les instruments de défense contre d'autres nations qui ont l'intention d'exploiter indûment l'espace territorial du Timor-Leste, en raison de la fragilité économique actuelle. ${ }^{[3]}$

Le Timor-Leste, jusqu'en 1975, appartenait au Portugal, ses colons. À partir de 1976, I'Indonésie l'envahit et commence à l'exploiter, et ce n'est qu'avec l'aide des Nations Unies (ONU) que ce pays a élu son premier président de la République en 2001, devenant un État indépendant et membre de l'ONU en 2002. Malgré son indépendance, le Timor-Leste a souffert de la tyrannie de l'Australie en ne recaladant pas les ressources financières pour l'exploitation de son territoire maritime. Le Portugal, l'Indonésie et l'Australie n'ont jamais investi correctement au Timor-Leste, bien qu'ils aient recueilli des millions d'argent provenant de l'extraction pétrolière dans cette région, ainsi que d'autres richesses. En raison de décennies de faibles investissements financiers, la nation timolaise doit se développer très politiquement et juridiquement afin de se produire dans le développement économique. ${ }^{[4]}$

L'objectif général de cet article scientifique est d'analyser le droit public international et pétrolier pour comprendre les raisons qui ont généré il y a plus de dix ans le conflit entre le Timor-Leste et l'Australie aux frontières maritimes. Pour comprendre le problème entourant le territoire maritime du Timor-Leste, plusieurs objectifs spécifiques ont été

a) présenter un aperçu, une vision politique, historique, juridique et économique du Timor-Leste et de ses relations avec l'Australie;

b) d'examiner le droit international relatif à la mer;

c) exploiter le droit au pétrole;

d) analyser le traité sur les frontières entre le Timor-Leste et l'Australie.

Cet article a son développement divisé en quatre parties: les possibilités d'évolution politico-sociale, historique, juridique et économique du Timor-Leste seront analysées tout au long de la recherche pour réaliser, analyser et vérifier si le traité entre L'Australie et le Timor-Leste contribueront en effet à l'avancement de la société 
timonoise. À partir de ces informations, il faudra comprendre les raisons qui ont conduit le peuple du Timor-Leste à avoir un faible niveau de développement dans plusieurs domaines.

Le premier chapitre traite des aspects historiques et géographiques, en plus de l'exploitation du Timor-Leste par d'autres nations, dans un contexte historique, de sorte que les raisons du contexte juridique, économique, politique et social actuel de ce pays soient comprises.

Dans le deuxième chapitre, les aspects sont explorés aux frontières de la mer du Timor-Leste et l'importance des relations internationales pour ce pays. Cette analyse a pour fonction d'observer les facteurs qui favorisent le peuple timorais à être à nouveau victime par les explorateurs, en raison de leur fragilité économique aujourd'hui. Pour comprendre les mécanismes de défense d'un État en matière d'attaques maritimes, il est nécessaire d'aborder le droit international de la mer. Cette partie du droit international traite des normes et principes convenus au niveau international liés à la propriété, à l'utilisation, à l'exploitation et à la protection de la mer et de ses ressources dans le monde entier. ${ }^{[5]}$

Dans le troisième chapitre, le concept et l'importance du pétrole et sa pertinence pour le développement des nations qui ont un immense espace territorial maritime et un petit espace terrestre, comme le Timor-Leste, seront explorés.

Le pétrole a été découvert au 19ème siècle et, depuis sa création, il ya eu d'importantes transformations dans l'humanité, C'est un mélange homogène de composés organiques, principalement des hydrocarbures, insolubles dans l'eau. Cette source d'énergie est également connue sous le nom de crue. Depuis 1859, il est considéré comme une préciosité et, au XXle siècle, il est très convoité pour le rôle pertinent qu'il joue dans le monde moderne. La recherche effrénée de l'Australie et de l'Indonésie pour le pouvoir et le développement économique a conduit à plusieurs conflits avec le Timor-Leste en raison de l'ambition de certains pays d'exploiter le territoire maritime qui ne leur appartenait pas. II est extrêmement important de comprendre comment l'extraction du pétrole se produit, ainsi que les ressources telles 
que le gaz naturel, pour comprendre les raisons qui ont conduit à une exploitation indue pendant des décennies de pétrole timorais. [6]

Par la suite, les obstacles au développement du Timor-Leste présents dans le quatrième chapitre. Les relations avec la communauté internationale sont extrêmement pertinentes pour le développement de ce pays. Les progrès dans le domaine juridique et politique sont essentiels à l'évolution économique. Les entreprises chargées d'exploiter les ressources naturelles du territoire maritime doivent négocier avec des professionnels timorais préparés et formés afin que les accords offrent de nombreux avantages au Timor-Leste. ${ }^{[7]}$

La présente étude n'a pas pour but d'épuiser toutes les questions, mais plutôt de stimuler les discussions sur le conflit entre le Timor-Leste et l'Australie. La technique de recherche utilisée dans ce travail consistera en la recherche bibliographique, à travers l'analyse des doctrines, des articles scientifiques, des bibliothèques virtuelles, ainsi que la recherche dans les textes juridiques à partir de sites Web reconnus comme des véhicules d'information accrédité par le gouvernement du Timor-Leste. La méthode adoptée sera le processus inductif par lequel l'étudiant, à travers l'étude de plusieurs positions d'endoctrineurs, partira de plusieurs compréhensions particulières de certains auteurs, pour parvenir à plusieurs conclusions générales. Une exploration bibliographique sera effectuée en relation avec le sujet, en particulier, avec chaque sous-chapitre du résumé pour parvenir à une conclusion générale du problème. ${ }^{[8]}$

\section{TIMOR-LESTE, GEOGRAPHY, HISTOIRE, ECONOMIE ET POLITIQUE}

La République démocratique du Timor-Leste est un pays côtier. Ses principaux produits économiques sont les ignames, le maïs, le café, la fumée, le caoutchouc, le bois de santal blanc, le bétail, les porcs, le buffle, entre autres. Ce pays est situé sur l'île du Timor et a des frontières avec l'Indonésie et l'Australie. Son territoire correspond à des parties de cette île, car en 1975, l'époque de l'indépendance du Portugal, l'autre moitié de l'archipel n'appartenait plus au domaine portugais. En raison de cette situation, il ya actuellement une partie de l'île qui est le territoire de l'Indonésie. 
Il est situé en Asie du Sud-Est, à la limite sud de l'archipel indonésien, au nord-ouest de l'Australie, près de l'Océanie. Au sud, il est de 250 à 400 milles marins à travers la mer du Timor avec le continent australien.

Le territoire maritime de ce pays, qui a une abondance de pétrole et de gaz naturel, a toujours attiré la cupidité des différents pays. Les traités et les invasions qui ont nui au timorais à diverses périodes historiques découlent du désir d'envahir et de prendre ses fonctions pour devenir une autorité légitime pour mener à bien l'exploitation de la mer du Timor-Leste. Afin d'obtenir un avantage, l'Australie a été le seul pays au monde à avoir officiellement reconnu l'annexion illégale du Timor-Leste par l'Indonésie dans les années 1970, bien qu'il y ait une résolution du Conseil de sécurité de l'Assemblée des Nations Unies Nations Unies qui ont condamné cette invasion. ${ }^{[9]}$

Figure 1 - Image caption Les frontières maritimes du Timor-Leste

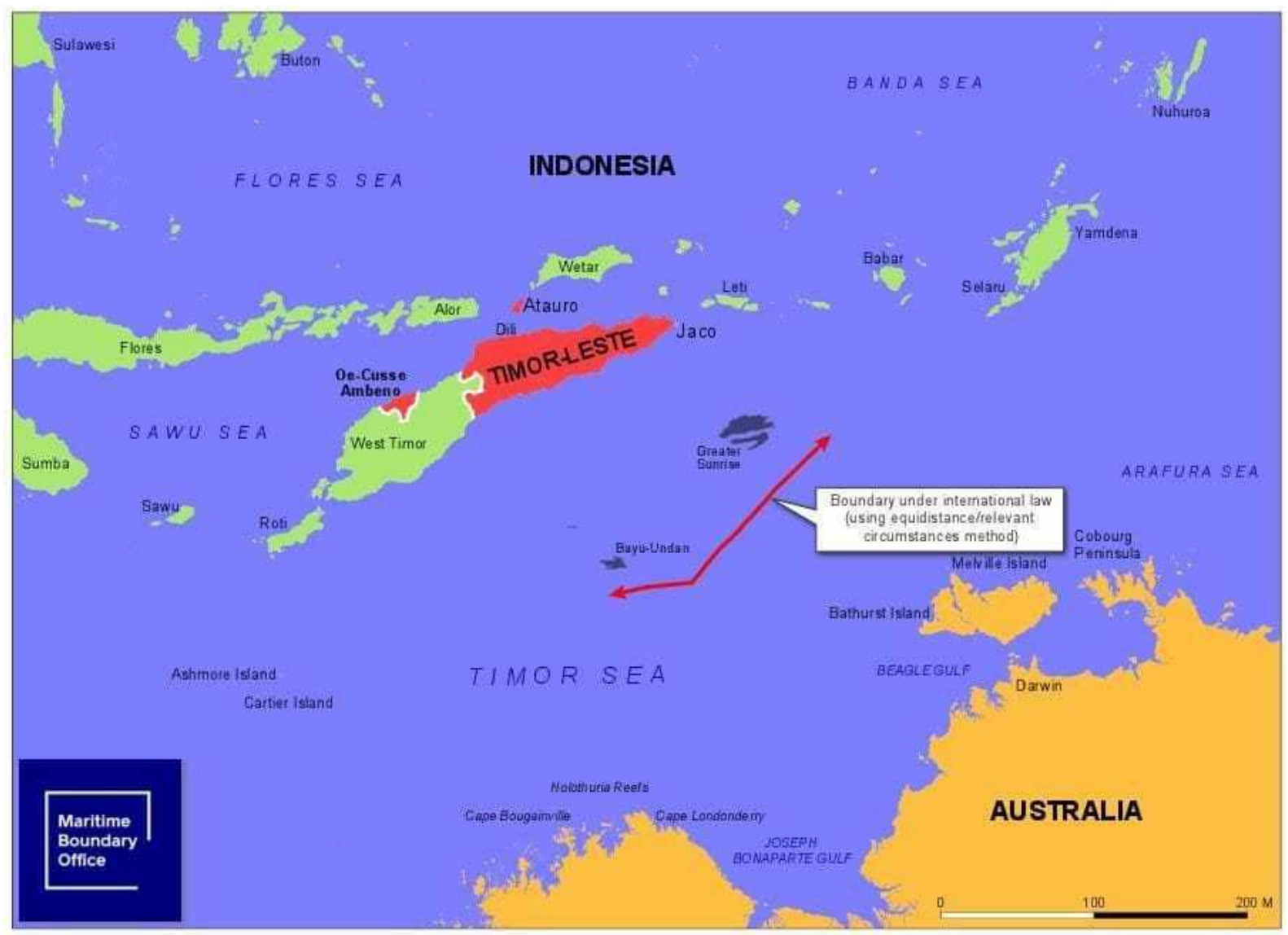


s (2018)Le Portugal, le Japon, l'Indonésie et l'Australie ont déjà été explorés à différentes époques. Le contexte historique de cette république est un facteur qui révèle les causes de la situation sociale, politique et économique actuelle de cette nation. Ce pays, qui a un petit territoire terrestre et environ un million d'habitants, a besoin de développer beaucoup dans plusieurs régions parce que c'est un pays jeune. Le Portugal a été le dominateur du Timor-Leste de 1515 à 1975 et a peu investi dans sa croissance. De 1942 à 1945, pendant la Seconde Guerre mondiale, il a été occupé par le Japon, mais bientôt les Portugais ont repris l'autorité de cette colonie. En avril 1975, un mouvement politique des forces armées, populairement connu sous le nom de 3D, a vu le jour au Portugal, dans le but de décoloniser, de démocratiser et de se développer. Pendant cette période, au Timor-Leste est né un mouvement révolutionnaire connu sous le nom de FRETILIN, Front révolutionnaire du Timor-Leste indépendant. Le 28 novembre 1975, son indépendance a eu lieu, entraînée par le Mouvement des timorais. L'indépendance n'a été observée que pendant dix jours. ${ }^{[11]}$

L'Indonésie, en juillet 1975, a envahi le Timor-Leste, restant environ vingt-six ans dans ce territoire, à une époque où de nombreux décès dus à la maladie et aux pénuries alimentaires se sont produits. La communauté internationale a été émue par le génocide qui s'y est produit. En 1999, l'ONU, avec le Portugal, a négocié un accord avec l'Indonésie, qui traitait de la possibilité d'organiser un référendum. En août 1999, les Timorais ont opté pour l'indépendance avec une majorité de voix, $78 \%$ de la population n'acceptant plus le massacre qu'ils ont subi. Mais les dominateurs n'acceptaient pas la libération. Des militaires indonésiens ont torturé et massacré le peuple. Beaucoup de Timorais ont fui vers la partie occidentale de l'île, tandis que tout ce qui a été construit le long de l'invasion a été détruit dans ce pays. Afin de contrôler le massacre, l'ONU a adopté la résolution 1246 d'août 1999 pour mettre en place une force multinationale pour stabiliser la situation. Après le retrait de l'Indonésie, le TimorLeste est devenu le bénéficiaire de l'aide de la communauté internationale, car il y avait des pertes humaines et des matériaux incalculables. Le 20 mai 2002, sans aide, l'indépendance de la République démocratique du Timor-Leste a été rétablie, observant le premier gouvernement démocratiquement élu. ${ }^{[12]}$ 
L'intérêt de l'Australie pour le Timor-Leste n'est pas nouveau. Les Australiens avaient des informations selon lesquelles dans la mer du Timor-Leste il y avait beaucoup de gaz et de pétrole. Depuis la découverte en 1960 qu'il y a de la richesse dans la mer du Timor, les gouvernements étrangers ont essayé de dominer l'île. Dans les années 1970, l'Australie était le seul pays qui reconnaissait que la moitié occidentale de l'île du Timor appartenait à I'Indonésie. II s'avère qu'en 1970, une société australienne appelée Woodside Petroleum a trouvé une énorme réserve de gaz dans la région connue sous le nom de The Sunrise and Troubadour Fields ou Greater Sunrise. ${ }^{[13]}$ L'intention de négocier pour des avantages territoriaux a été l'une des raisons pour lesquelles les Australiens ont ignoré l'opinion négative de la communauté internationale, qui n'était pas d'accord avec la conduite de l'Indonésie d'envahir la partie occidentale de l'île du Timor. Intéressés à tirer profit de la découverte de richesses dans la mer du Timor-Leste, l'Indonésie et l'Australie ont signé le traité. Ce document concernait les frontières maritimes entre les deux pays, mais l'Australie a pris le territoire où se trouvaient les réserves de gaz. Insatisfaite du traité qu'elle a signé en 1975, l'Indonésie a envahi l'Australie pour obtenir un pourcentage sur l'exploitation des ressources gazières dans cette région. Les Timorais n'étaient pas d'accord avec le traité qui partageait la richesse de leur territoire entre l'Indonésie et l'Australie, mais les deux nations étrangères impliquées dans l'accord international ont ignoré la position du monde, contrairement à leur pacte. Le 20 mai 2002, date du rétablissement de l'indépendance du Timor, le pays a cherché à prendre possession du territoire qui y avait droit. Le Timor n'appartenait plus à l'Indonésie. Les traités qui avaient été conclus avant l'indépendance n'avaient plus de valeur. ${ }^{[14]}$

L'Australie, en 2002, dans le but de rester dominant le territoire maritime qui appartiendrait au Timor-Leste, s'est retirée de toutes les procédures frontalières contraignantes dont elle faisait partie. En 2006, il y a eu un traité entre l'Australie et le Timor-Leste sur certains ajustements maritimes dans la mer du Timor, un accord qui a déplu aux timorais parce qu'il a permis d'éviter des changements dans les négociations juridiques ou les actions en justice pendant cinquante ans. C'est en 2016 que les personnes qui se sont senties lésées par le traité ont informé les Australiens qu'il résoudrait le problème des frontières maritimes par une conciliation obligatoire 
prévue par la Convention NATIONS UNIES, l'article 298 et l'annexe V, qui traite de Droits de la mer. ${ }^{[15]}$

La domination du Timor-Leste par d'autres nations, à différentes périodes historiques, lui a grandement nui. Plusieurs réflexions sur l'exploitation de l'Indonésie et de l'Australie se font sentir dans le développement du peuple timorais dans divers domaines. Depuis 2002, à la veille de l'indépendance du Timor-Leste, les Australiens se sont retirés de plusieurs traités internationaux pour empêcher la perte d'exploitation du territoire non maritime. Les Australiens et les Timorais ont des territoires séparés pour moins de 400 milles marins. En raison de la proximité entre les deux nations, leurs territoires maritimes doivent être définis d'une manière particulière et spécifique, selon le principe d'équité. II est essentiel d'approfondir les connaissances sur le droit de la mer et du pétrole pour comprendre les raisons de fait et de droit qui légitiment la lutte du peuple timorais pour son territoire maritime. ${ }^{[16]}$

\section{DROIT DE LA MER ET DU PÉTROLE}

Le droit de la mer appartient au droit international, qui observe la souveraineté et la compétence des États, définissant l'étendue de leur domaine maritime. II réglemente également plusieurs autres sujets tels que l'exploitation des ressources existantes sur le lit et sur le fond marin, en plus de la préservation et la conservation de l'environnement marin. ${ }^{[17]}$ L'une des plus grandes richesses trouvées au fond de la mer du Timor est le pétrole, qui se distingue par être un liquide noir visqueux et inflammable, moins dense que l'eau. II se compose d'un mélange d'hydrocarbures, de molécules composées d'atomes de carbone et d'hydrogène, ainsi que de molécules de soufre, d'azote, d'oxygène et d'ions métalliques, et est situé dans des réservoirs souterrains naturels. ${ }^{[18]}$

La Conférence sur les droits maritimes a eu lieu à Genève en 1958. En 1969, il a été ratifié par presque tous les pays du monde. II traite de divers sujets ou sujets qui traitent de la connexion étroite dans l'espace océanique entre les nations. La Convention des Nations Unies sur les droits de la mer (UNCLOS) de 1982 a été signée à Montego Bay, en Jamaïque, bien qu'elle soit apparue à la conférence de Genève. 
Dans ce document sont les règles et les principes de la mer territoriale, cont ${ }^{[19]}$ igus, économiques et continentaux. ${ }^{[20]}$ En ce qui concerne la souveraineté de l'État côtier sur les eaux adjacentes ${ }^{[21]}$, il réglemente tous les pays qui l'ont adoptée, acceptée ou ratifiée. II existe également des normes connexes en matière de gestion des ressources marines et de lutte contre la pollution. ${ }^{[22]}$

Le PNUD détermine que la mer de la côte a 12 miles nautiques, c'est-à-dire $22 \mathrm{~km}$. Au plan vertical, il s'étend à l'espace aérien, l'ayant comme limite. L'eau de mer de la surface est limitée par le contact avec le fond marin, le sol, ainsi que tout sous-sol est régi par le régime juridique territorial de la mer. ${ }^{[23]}$ Le droit de la mer aux États côtiers était garanti une exploitation économique exclusive dans un air de 200 milles marins, mais cette règle ne s'applique pas aux États qui sont à moins de 400 milles de leurs zones contigus. ${ }^{[24]}$

Figure 2 - Mer territoriale

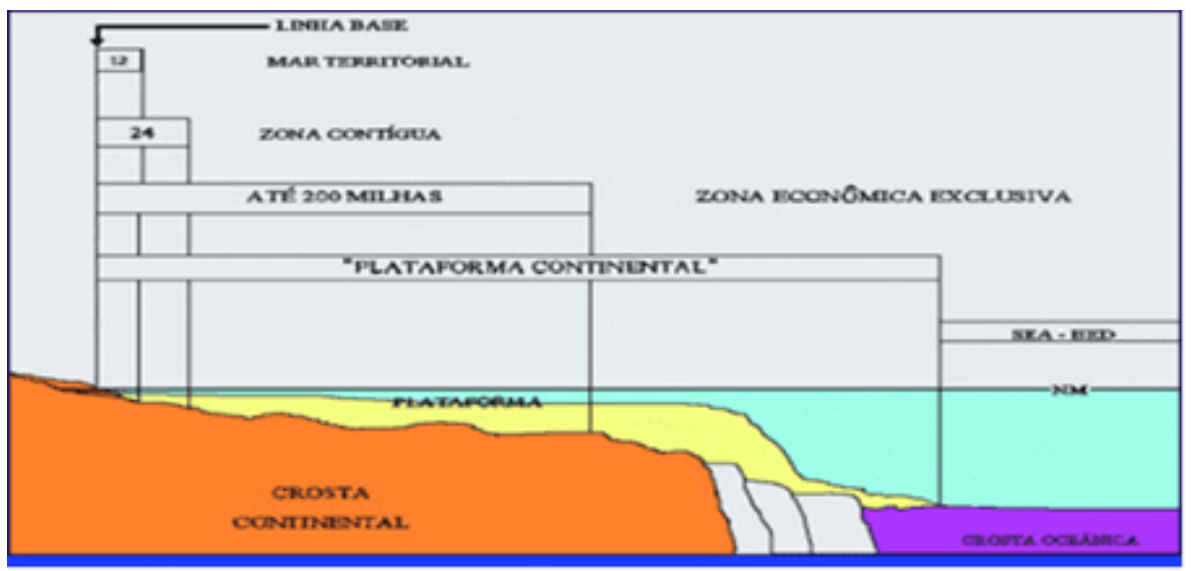

La mer territoriale est la zone située entre les eaux intérieures et la haute mer. C'est le maintien de la souveraineté d'un pays côtier qui dépasse son territoire et ses eaux, selon les articles 2 et 3 de l'UNCLOS.[25] L'État exerce sa souveraineté sur sa mer territoriale, son espace aérien, ainsi que sur le lit et le sous-sol sous la mer territoriale, selon Art. 02 à 32 de la Convention relative aux droits de la mer. ${ }^{[26]}$ La frontière extérieure de la mer territoriale de chaque nation est de vingt-deux kilomètres. L'État exerce sa compétence sur les activités d'intérêt national dans la zone maritime adjacente, qui est fixée à 12 miles. La Convention des Nations Unies a créé la Zone 
économique exclusive, la ZEE, pour équilibrer les intérêts entre les pays. Il s'agit d'une zone située au-delà de la mer Territoriale. II se réfère à la zone près de la zone contigue et s'étend jusqu'à 200 miles de la côte. L'État a le droit de naviguer, de survoler, d'installer des câbles et des conduits marins, ainsi que d'exploiter les minéraux présents dans le sol et le sous-sol marin. Le Timor-Leste et l'Australie sont séparés à moins de 400 milles de distance, et pour cette raison cette règle ne résout pas les conflits au-dessus des frontières entre les deux nations. ${ }^{[27]}$

Les Timorais et les Australiens sont des peuples appartenant à des États avec des côtes adjacentes ou situées face à face. Ils ne respectent pas les limites fixées par la Convention des Nations Unies pour l'application des droits de la mer, liées à la souveraineté de l'État dans la mer territoriale. La distance de la côte côtière entre les pays est de 300 miles. Ces pays doivent délimiter les frontières maritimes par accord, tel que déterminé par les règles du droit international. Les nations doivent parvenir à une solution juste, juste et équivalente. ${ }^{[28]}$

La Convention de Genève a des critères de délimitation parallèle. La méthode de ligne médiane est utilisée dans des circonstances spéciales pour les états avec des dos opposés. II réglemente un réajustement de la ligne médiane entre les pays. La Convention sur le droit de la mer stipule que, compte tenu de l'absence d'une distance de 400 milles pour la séparation entre deux pays, le principe de l'équidistance doit être utilisé. [29]

L'industrie pétrolière est extrêmement importante. La société d'aujourd'hui en dépend et de ses dérivés. II s'agit d'un combustible fossile, une source d'énergie électrique pour la plupart des pays développés et en développement. II est essentiel pour la fabrication d'une gamme de produits tels que le diesel, le kérosène et l'essence. Il est également présent dans les intrants et dans l'industrie pétrochimique, à travers la paraffine et le naphta. Beaucoup de médicaments ont dans leur composition des dérivés du pétrole. Il est impossible de réfléchir sur les moisissures actuelles de la vie humaine sans asphalte, plastiques et aspirine. L'huile peut être observée dans les carburants utilisés pour la locomotion des gens. II est contenu dans une multitude de produits chimiques et pétrochimiques fondamentaux pour le développement d'une 
nation. En raison de sa vaste applicabilité et parce qu'il est une source d'énergie épuisante, les Australiens ont ignoré le droit international de la mer depuis de nombreuses années. Ils se sont retirés du PNUD pour empêcher le peuple timorais d'avoir droit à des profits sur l'exploitation des compagnies pétrolières Bayu Undan et Greater Sunrise. ${ }^{[30]}$

En raison de l'importance du pétrole, l'Organisation des pays exportateurs de pétrole (OPEP) a émergé à Vienne, autriche en 1960. Les nations fondatrices étaient la République islamique d'Iran, l'Irak, le Koweït, l'Arabie saoudite et le Venezuela. L'objectif de l'Opec est d'établir une politique commune pour le pétrole, en protégeant les revenus des pays producteurs. Avant la création de cette organisation, les pays exportateurs de pétrole qui détenaient la plupart des réserves pétrolières de l'époque n'en bénéficiaient guère. Les grandes sociétés réglementaient le marché pétrolier international. La valeur des combustibles fossiles versés aux pays producteurs et à la revente au consommateur final était contrôlée par les compagnies pétrolières. Les sociétés américaines Exxon, Texaco, Amoco et Chevro, ainsi que les néerlandais Royal Dutch Shell et British Petroleum s'appelaient Seven Sisters. Ils ont effectué l'exploration, le raffinage, le transport et la revente du pétrole, mais seule une petite partie des fruits de l'extraction était destinée aux États producteurs. À l'heure actuelle, les Nations Unies observent également les actions des compagnies pétrolières afin qu'un accord approprié puisse être conclu entre les États producteurs et les sociétés extracteurs de pétrole. ${ }^{[31]}$

Le Timor-Leste est exploré depuis des décennies par l'Australie. Cette conduite a été largement motivée par des raisons économiques, en raison de l'intérêt pour la richesse du territoire marin timorais. II n'est pas nouveau que l'industrie pétrolière soit motivée à retirer l'Australie de la communauté internationale. Les Australiens n'ont pas été disposés à traiter des questions liées aux droits de la mer. L'intérêt pour les bénéfices pour l'extraction du pétrole et du gaz naturel de la région appartenant au Timor-Leste y a empêché de parvenir à un accord entre les deux pays pendant de nombreuses années. ${ }^{[32]}$ 
Les Timorais ont été lésés par l'Australie depuis l'indépendance en 2002. Toutes les réserves de pétrole et de gaz se trouvent du côté timorais de la ligne médiane, c'està-dire plus près du Timor-Leste que du territoire australien. L'Australie ne reconnaît plus la compétence de la Cour internationale de Justice et de la Convention des Nations Unies sur le droit de la mer pour délimiter ses frontières sur la ligne médiane avec le Timor-Leste quelques mois avant son indépendance. La suppression de l'Australie du PNUD avait l'intention d'éviter la perte ou la réduction de l'espace territorial maritime, car elle appartenait au droit aux Timorais. La Convention des Nations Unies relative aux droits de la mer stipule que chaque pays doit se délimiter en tant que zone économique exclusive à 200 milles marins, à partir de la limite extérieure de sa mer territoriale, à 12 milles de la côte. Le PNUD traite également des grandes profondeurs de la mer qui sont connues sous le nom de zone ou zone $A$, selon l'art. 1. Cette zone est composée de lit marin et océanique et de leur sous-sol. [33]

Figure 3 - Ligne de démarcation frontalière de l'Australie

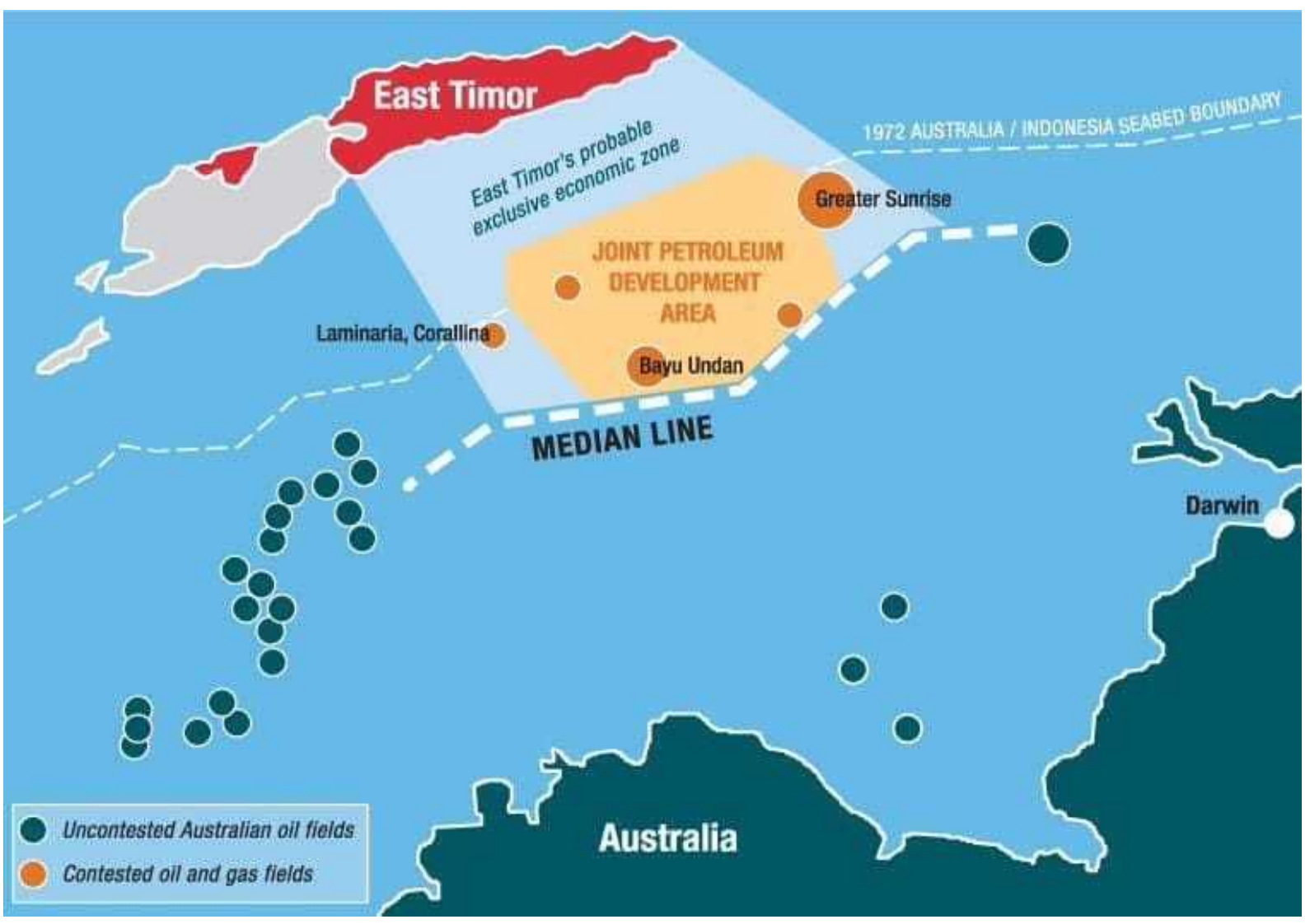


Les principes contenus dans la Convention des Nations Unies relative aux droits de la mer stipulent que la frontière maritime entre les deux pays concernés doit être définie par la ligne équidistante, c'est-à-dire la ligne médiane. La délimitation des frontières entre l'Australie et le Timor-Leste par le principe de l'équidistance comprend le territoire timorais avec toute la zone d'exploration du puits de la compagnie pétrolière Bayu Undan. Le champ pétrolifère du Grand Lever du Soleil est situé dans la zone dite de la zone de coopération et, selon l'UNCLOS, ce territoire appartient également au peuple timorais. ${ }^{[35]}$

Le pétrole est une richesse qui se trouve en abondance au Timor-Leste. La ligne médiée doit être tracée pour définir l'espace maritime entre deux pays qui se séparent pour moins de 400 milles marins. Des réservoirs de gaz naturel et de pétrole ont été découverts par des entreprises australiennes au Timor-Leste, avant leur indépendance. Pour cette raison, les Australiens ont eu beaucoup de résistance à un traité définitif sur le droit à l'exploitation de la mer du Timor. Les difficultés rencontrées par ces personnes pour obtenir leurs droits maritimes sont des questions importantes. L'analyse des détails qui ont précédé le traité définitif de 2018, qui traite des frontières du Timor, est essentielle pour comprendre la proportion d'évolutions que signifie ce pacte. ${ }^{[36]}$

\section{TRAITÉ ENTRE L’AUSTRALIE ET LE TIMOR-LESTE}

En 2002, le Timor-Leste a obtenu son indépendance de l'Indonésie, mais depuis cette année-là, les frontières maritimes définitives du Timore n'ont pas été délimitées. Pendant de nombreuses années, les Australiens n'ont aucun intérêt à traiter avec les frontières maritimes de timor-leste, en respectant les normes du droit international. Ils avaient l'intention de continuer à utiliser les ressources de la mer qui ne leur appartenaient pas. Bien qu'ils aient longtemps causé des dommages au développement du Timor-Leste dans plusieurs régions, le pacte n'a pas prévu l'indemnisation des dommages causés depuis 2002. ${ }^{[37]}$

Le peuple timorais ne sera pas remboursé pour les dommages causés à leur évolution dans les domaines de l'éducation, sociale, économique, politique, entre autres qui 
nécessitent des ressources financières minimales pour se produire. Mais lorsque le pacte a été mis en œuvre, il a été observé que le Timor-Leste a été considérablement lésé au fil des ans par l'Australie. ${ }^{[38]}$ Afin d'encourager le développement, industriel, technologique et économique du timorais, il a été convenu que l'entreprise qui réaliserait l'exploration du Grand Lever de soleil devrait embaucher les citoyens du Timor-Leste, ce qui faciliterait la formation encourager le développement de leurs études, ainsi qu'il aurait le devoir d'adopter le pays comme première option pour l'acquisition de biens et de services. ${ }^{[39]}$

Afin d'éviter les pertes économiques, l'Australie s'est retirée de plusieurs traités internationaux dont elle faisait partie intégrante des mois précédant l'indépendance du Timor-Leste. ${ }^{[40]}$ Afin d'éviter que l'un ou l'autre pays ne respecte le pacte à l'avenir, les nations ont stipulé que le traité ne serait pas soumis à un droit unilatéral à la dénonciation, au retrait ou à la suspension. Dans le texte du document, il a été précisé que toutes les clauses exprimées dans le traité et les annexes font partie du pacte et ne peuvent être ignorées ou mises en évidence. Face à un éventuel différend futur, il a été convenu qu'il pourrait être résolu avec l'aide des membres de la Commission de conciliation qui ont négocié le pacte entre les deux nations en 2018. Toutefois, dans la persistance du désaccord sur les questions, les questions abordées avec l'intervention des membres du Comité de conciliation pourraient être portées devant le tribunal arbitral, et cette deuxième décision aurait un effet contraignant. ${ }^{[41]}$

Le 6 mars 2018, l'accord entre le Timor-Leste et l'Australie a eu lieu. Ce pacte délimite le bassin continental. Dans ce document, la ligne médiane et les frontières latérales ont été délimitées à l'est et à l'ouest de l'ancienne zone connue sous le nom de Timor Gap, selon l'art. 1er du traité sur la frontière maritime de Timor-leste. ${ }^{[42]}$ Dans ce nouveau contexte, le Timor-Leste est autorisé à exploiter les ressources des sols marins tels que le pétrole et le gaz naturel, en plus de fournir les avantages aux droits de la zone économique exclusive. Cette nation a également obtenu le droit d'exploiter d'autres ressources maritimes telles que la pêche, ce qui a permis d'accroître les ressources financières du pays. ${ }^{[43]}$ 
Figure 4 - Traité d'Australie et Timor-Leste aux frontières du Timor

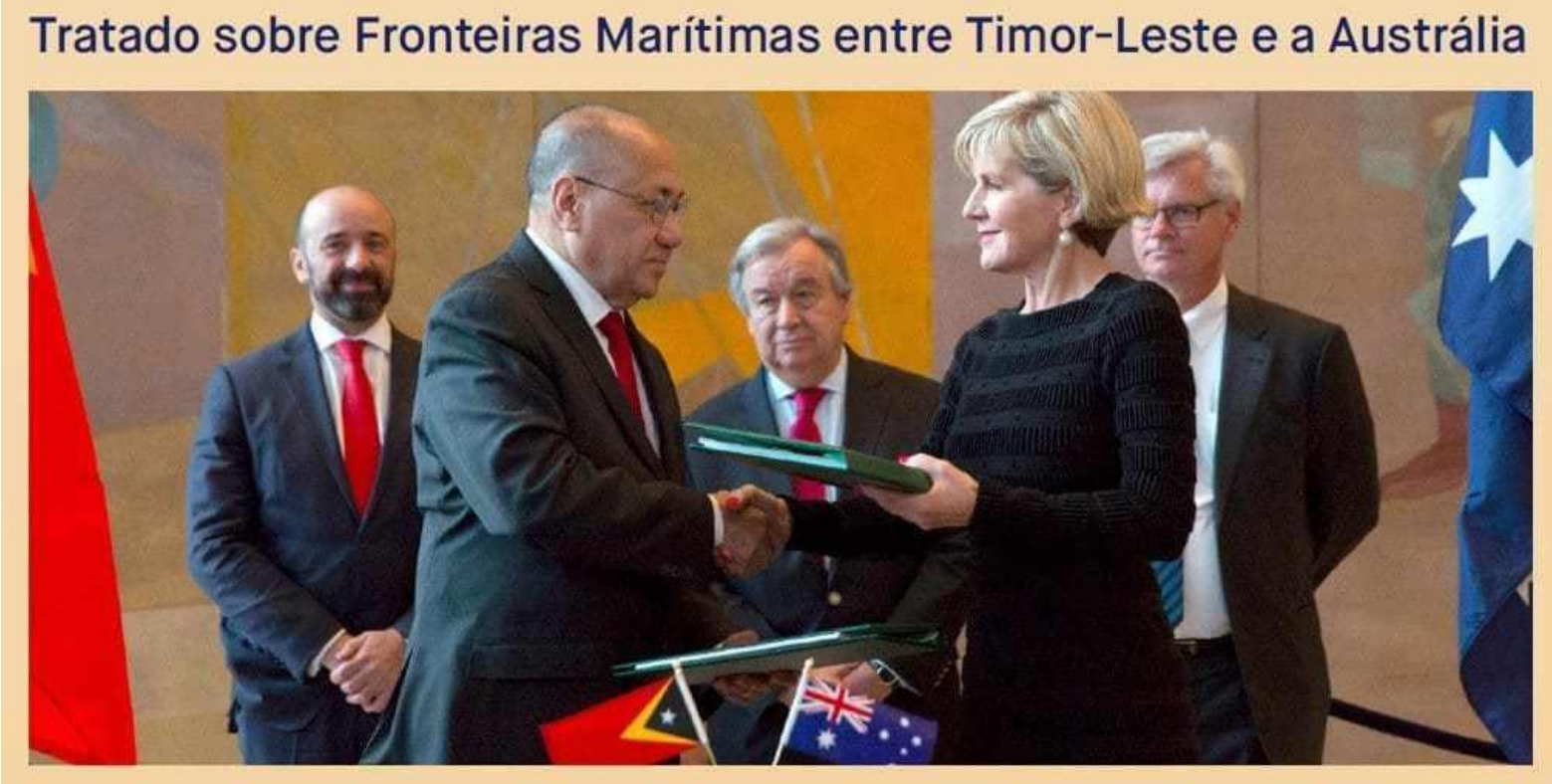

Le Timor-Leste et l'Australie ont assoupli leurs intérêts pour résoudre les désaccords qui les empêchaient de s'entendre. Dans la zone territoriale maritime où se trouve le Grand Lever de soleil, il a été convenu qu'elle appartiendrait à chacun des pays impliqués dans l'accord à la moitié des résultats de l'exploitation de cette région territoriale maritime. Les camps de Bayu-Undan, Buffalo et Kitan ont entièrement intégré le territoire timorais. ${ }^{[45]}$

Les modalités de partage des recettes entre le Timor-Leste et l'Australie n'ont pas été définies, car elles dépendraient de plusieurs facteurs. Plus la capacité de développer des techniques et de les appliquer est grande, plus leurs profits seraient élevés. Selon les moyens d'explorer les champs du Grand Lever de soleil, les résultats seraient partagés. Au cours de l'année où le pacte a été conclu, il a été convenu que les revenus des champs pétrolifères appartiendraient à $30 \%$ à l'Australie et à $70 \%$ au Timor-Leste s'il y avait une augmentation de l'exploration par le développement d'un gazoduc pour le peuple timorais. II a également été prévu que, dans le cas où les champs du Grand Lever de soleil seraient développés par le biais d'un pipeline vers l'Australie, les pourcentages seraient modifiés, appartenant à $20 \%$ pour les Australiens et $80 \%$ pour les Timorais. ${ }^{[46]}$ 
Dans le traité définitif sur les frontières, selon l'art. 12 du document juridique, il a été défini que l'exploration du gaz et du pétrole dans la région du champ Greater Sunrise dépendrait de la définition entre les parties du plan de développement. II s'avère qu'après le début des activités d'exploration de la région maritime qui auraient partagé les revenus, la société sous contrat se soumettrait à la juridiction exclusive du pays où elle est située. Le Conseil de surveillance de deux représentants du Timor-Leste et d'un représentant de l'Australie serait créé. Afin de trouver des solutions aux litiges, il y aurait un comité indépendant de résolution des conflits pour les décisions sur les stratégies adoptées pour l'exploration du champ pétrolier mentionné ci-dessus. ${ }^{[4]}$

Pendant des décennies, l'Australie a démontré son intention de tirer pleinement profit de l'exploitation des champs pétrolifères. Une intervention internationale était nécessaire pour réduire le conflit sur les frontières entre Timorais et Australiens. Le traité définitif sur les frontières traite de la ligne médiane et des limites latérales sur le territoire du Timor-Leste et partage les revenus d'une région pétrolière entre les pays concernés. Mais ce pacte n'éteint pas la possibilité de conflits futurs sur les bénéfices des champs du Grand Lever de soleil. De nombreuses questions étaient en attente de clarification, exigeant des conflits concrets pour que les positions soient adoptées. Face à cette nouvelle situation, les Timorais devront faire face à plusieurs obstacles afin de pouvoir profiter de toutes les possibilités de leur développement dans différents domaines. ${ }^{[48]}$

\section{LES OBSTACLES AU DÉVELOPPEMENT DU TIMOR-LESTE}

Le Timor-Leste est un État maritime et pas seulement du pétrole. II existe plusieurs secteurs qui peuvent contribuer à l'évolution de la nation. Pendant de nombreuses années, il a été exploré par le Portugal, l'Indonésie et l'Australie. Peu de ressources ont été investies dans l'éducation et l'infrastructure. La nation a été blessée dans le développement de plusieurs domaines en raison de la négligence de l'Indonésie. II y a peu de professionnels ayant des compétences techniques, politiques, juridiques et administratives, car pendant la période de domination du peuple, seuls des professionnels étrangers ont été embauchés pour effectuer des activités dans ces domaines. ${ }^{[49]}$ 
Figure 5 - Élèves d’une école de la périphérie timolaise

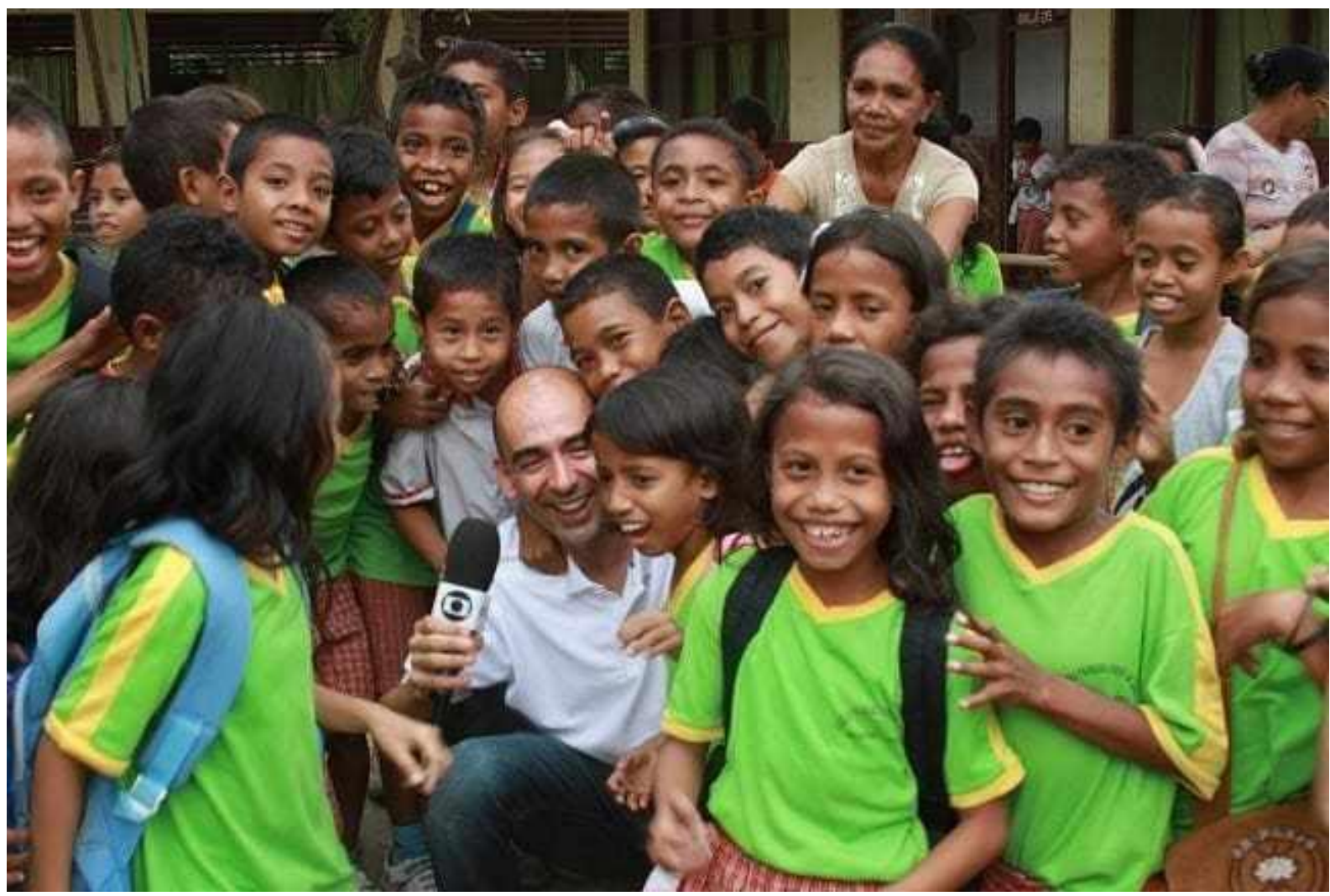

011). Depuis 2011, le Timor-Leste a un plan de développement stratégique qui comprend l'évolution du capital humain, des infrastructures, de l'économie, de la politique et du domaine administratif. L'objectif du gouvernement timorais est d'atteindre les objectifs énoncés dans le plan de développement d'ici 2030. Les ressources provenant des droits d'exploitation de son territoire maritime accéléreront la mise en œuvre des objectifs proposés dans le plan de développement timorais. Le pacte de l'Australie sur les frontières définitives va certainement générer plusieurs situations particulières et sans précédent. Des connaissances spécifiques sur les méthodes d'exploration du pétrole et du gaz naturel seront essentielles pour comprendre quelles solutions de rechange apporteront le plus de profits au pays. Après la ratification du traité, de nombreuses normes ont été établies afin que les timorais puissent jouir de leurs droits, mais auront besoin de professionnels qualifiés dans divers domaines. Et l'un des principaux défis auxquels le Timor-Leste est confronté est la courte expérience de l'administration publique, avec de grands 
volumes de recettes financières, qui se multiplieront en raison du traité définitif sur ses frontières. ${ }^{[51]}$

Dans le document du traité, des normes impliquant de grandes compagnies internationales d'explorateurs ont été établies. Les compagnies pétrolières devraient contribuer au développement de timor-leste en embauchant de préférence des professionnels, des services et des produits dans le pays. Les Timorais se rapporteront non seulement aux représentants du gouvernement australien, mais avec le résumé expérimenté d'initiative privée. Les représentants étrangers du gouvernement australien ou des entreprises privées de divers pays peuvent avoir une grande capacité de négocier et de traiter avec les Timorais inexpérimentés. Le TimorLeste a des professionnels avec peu de compétences techniques, mais cette situation ne devrait pas compromettre le développement. Ces entrepreneurs auront pour mission d'aider à l'éducation et à l'enseignement des compétences et des connaissances requises des travailleurs pour effectuer des tâches liées aux activités d'exploration. L'augmentation des ressources financières contribuera à la transformation des secteurs, techniques, administratifs, politiques, juridiques, entre autres, facilitant la transposition des obstacles au développement de la nation. ${ }^{[52]}$

L'exploration du gaz et du pétrole exigera que le pouvoir législatif du Timor-Leste soit conscient de l'émergence de faits nouveaux découlant de cette activité. Les organismes d'État devront trouver des réponses rapides aux divergences entre l'État, les représentants des sociétés d'exploitation et l'Australie. Bien que la société sous contrat ait l'obligation de se soumettre à la juridiction exclusive du pays où elle se trouve, l'expérience et la capacité des représentants du secteur privé de manipuler les négociations devraient être prises en considération. II est indéniable que le gouvernement du Timor-Leste adopte des positions qui contribuent au progrès dans diverses régions du pays, mais il est un fait que les pouvoirs exécutif, législatif et judiciaire timorais sont encore en train de se développer, parce que c'est un pays très jeune. ${ }^{[53]}$

Le Timor-Leste a une vaste expérience dans les combats, comme le peuple organisé contre l'invasion de l'Indonésie et a obtenu leur indépendance. ${ }^{[54]}$ À I'heure actuelle, il 
progresse dans la recherche de l'éducation, de l'infrastructure, de l'économie, des politiques et d'une gestion administrative adéquate pour les citoyens. Dans le pacte sur les frontières définitives, la situation de fragilité du Timor-Leste a été reconnue. Et les mécanismes pour aider à l'évolution du pays ont été positifs dans l'accord. ${ }^{[55]}$

Le Gouvernement du Timor-Leste aura des tâches complexes, et un secteur juridique et administratif prêt à agir dans de nouvelles activités sera nécessaire. Des rapports et des plans d'aménagement sur l'exploration des champs de Bayu-Undan, Buffalo et Kitan devront être publiés pendant la période de transition de l'exploration de cette région. Ce droit exigera des connaissances techniques spécifiques sur l'exploration pétrolière et gazière. ${ }^{[56]}$ De nombreux secteurs sont liés au développement d'une nation. Les politiques d'investissement dans les secteurs qui ont des sources d'exploitation renouvelables doivent être observées par le Gouvernement du TimorLeste. Les dispositions du Pacte sur les frontières timonoises, liées au devoir des entreprises d'aider à l'évolution des différents secteurs, devraient être prises en considération. L'État devra s'efforcer de développer dans la même mesure qu'il devrait exiger une initiative privée pour contribuer à l'évolution du pays. ${ }^{[57]}$

La croissance économique est essentielle aux progrès réalisés dans les domaines de l'infrastructure, des politiques et de l'administration. L'essor économique du TimorLeste est lié aux secteurs de l'agriculture, du tourisme et de la mer et pas seulement à l'exploration pétrolière et gazière. L'agriculture est essentielle pour le Timor-Leste, ainsi qu'une grande pertinence pour plusieurs autres pays. De là, la nourriture est produite. Les produits primaires issus de l'agriculture peuvent être utilisés par les industries, le commerce et le secteur des services. Ils peuvent devenir la base du maintien de l'économie nationale et internationale. ${ }^{[58]}$

L'exploration de l'économie maritime est également cruciale pour l'évolution de la nation timolaise. Beaucoup de gens dépendent de la mer et se soutiennent avec les ressources générées par la pêche et la récolte des espèces marines. II s'avère que, dans le territoire maritime de ce pays, il y a un passage naturel de poissons. Et à travers les développements apportés par le pacte définitif sur les frontières, plusieurs espèces de poissons pourraient être exploitées par les industries nationales. En plus 
de l'augmentation des revenus pour l'émergence d'industries intéressées par les nombreuses espèces d'écoles que cette région a, cette nation est située dans le triangle corallien. II s'agit d'un patrimoine culturel qui peut être apprécié par le tourisme, ainsi qu'il peut servir d'intérêt à la recherche scientifique. Les caractéristiques cidessus peuvent générer l'augmentation directe et indirecte des ressources pour l'État, à condition que des mesures administratives appropriées soient prises. ${ }^{[59]}$

L'infrastructure des ports et aéroports timorais est également fondamentale pour la croissance des pays. Les paramètres des constructions stratégiques doivent surveiller les besoins de navigation du scénario global selon lequel le territoire timorais pourrait transiter par le territoire timorais. Les ports de Dili, Oe-cusse, Hera, Caravela et Com ont actuellement des caractéristiques insuffisantes, ce qui nécessite des investissements à considérer comme des centres industriels. Cette évolution créera des opportunités pour le Timor-Leste de devenir un transit de marchandises de pétroliers, en plus d'en faire une nation possédant un grand centre industriel qui relie le Pacifique et les océans Indien. ${ }^{60]}$

Le Timor-Leste doit continuer à progresser dans de nombreux domaines. Les secteurs agricole, pétrolier, maritime, touristique, éducatif, juridique, politique et économique ont besoin de transformations. II existe déjà un plan de politique de développement qui a été créé en 2011. Cependant, il est nécessaire de l'adapter à la nouvelle réalité dans laquelle se trouve le pays, après les changements qui ont eu lieu par la définition de ses frontières maritimes finales. ${ }^{[61]}$ Des stratégies spécifiques pour chaque secteur devront être retracées au niveau national interne. Et, sur la base de ces nouveaux objectifs, les positions des sociétés d'exploration pétrolière et gazière devraient être adoptées, tout comme toutes les décisions de l'Etat seront afin d'atteindre ces objectifs de croissance. ${ }^{[62]}$

\section{CONCLUSION}

Le Timor-Leste est situé en Asie du Sud-Est et est un pays très jeune, qui a obtenu son indépendance en 2002. C'est le voisin de l'Indonésie, qui se trouve au nord, et au sud est l'Australie. II a une nation caractérisée par sa force et sa lutte. Ce guerrier et 
les gens souffrants communiquent par la langue portugaise et le Tetum, officiellement, mais sur le territoire il y a plus de 14 langues indigènes. II a été colonisé par le Portugal et envahi par l'Indonésie en 1975. II a un trésor situé dans son territoire maritime, en raison du pétrole et du gaz naturel qui dans la mer du Timor se rencontrent. Ces richesses du Timor-Leste ont fait l'objet d'une bataille avec l'Australie. Le désaccord entre les Australiens et les Timorais a commencé des mois avant l'indépendance du Timor-Leste de l'Indonésie. L'Australie n'avait aucun intérêt à négocier, comme le prévoit le droit international de la mer, pour éviter la perte de recettes provenant de la mer du Timor. Mais en 2018, un traité définitif sur les frontières du Timor-Leste a été conclu avec les Australiens. Ce fut le moment historique où la nation a obtenu la reconnaissance du droit de jouir de sa mer territoriale.

La situation économique délicate du pays a été reconnue dans le traité frontalier définitif du Timor-Leste. La rareté des ressources économiques est liée à l'exploitation de cette nation d'une manière dévastatrice. Le Portugal a peu investi au cours de la période de plus de 400 ans au cours de laquelle ce pays était sa colonie. Après avoir été libéré de la domination portugaise, une nouvelle attaque contre le Timor-Leste a eu lieu. Pendant plus de vingt-quatre ans, les timorais ont souffert de l'occupation de I'Indonésie, mais de petits investissements ont été faits. Cependant, à partir de la période où le peuple timorais a montré son intérêt pour la lutte pour son indépendance, tout a été détruit par le peuple indonésien. Dans la phase initiale de l'occupation du Timor-Leste par l'Indonésie, la communauté internationale a contribué à la reconstruction fondamentale du pays.

L'indépendance des Timorais ne leur donne pas le droit immédiat de prendre possession de la richesse maritime qui leur appartenait, en raison du fait qu'ils étaient sur leur territoire. Des accords intérimaires sur les frontières, des pactes qui nuisaient au timorais ont été conclus avec l'Australie avant 2018. Depuis 2002, le Timor-Leste revendique la propriété de son territoire maritime, comme le prévoit les normes de droit international, UNCLOS/1982. II s'avère que quelques mois avant l'indépendance du Timor-Leste, l'Australie s'est retirée de plusieurs pactes internationaux. 
Étant donné que les territoires maritimes du Timor-Leste et de l'Australie se sont séparés par une distance de moins de 400 milles marins, une courte distance entre les territoires des deux nations, la ligne de délimitation des frontières adoptée a été définie par la norme de equidistance et ligne médiane. C'est le modèle prévu par la Convention des Nations Unies relative aux droits de la mer de 1982, utilisée pour résoudre les conflits qui ont été sur des territoires qui se distancent sur moins de 400 milles marins.

Les Australiens ont évité de parvenir depuis de nombreuses années à un accord avec les timorais sur les frontières définitives, conformément aux règles de la Convention des Nations Unies relative aux droits de la mer. Le but de cette position était de manquer de respect à la manière de délimiter les frontières maritimes, qui prévoit comme norme l'adoption de la ligne médiane et l'équidistance dans des conflits spécifiques sur la définition des frontières. La raison du retrait de l'Australie de la communauté internationale est liée à l'extension du profit de l'exploitation de la mer qui appartient au peuple timorais.

Après plusieurs tentatives d'accord définitif sur les frontières du Timor avec l'Australie, le pays a réussi. De toute évidence et plus précisément, dans un document qui contient plus de 11 articles, les frontières maritimes bilatérales permanentes ont été abordées le long d'une ligne essentiellement équidistante entre les deux nations. Le Timor-Leste a vu ses frontières délimitées comme le prévoit la Convention des Nations Unies sur le droit de la mer. Il a été convenu dans le traité qu'il existe un lien indivisible entre la frontière maritime du Timor-Leste et l'Australie. On a reconnu l'existence d'une zone maritime appartenant aux deux nations impliquées dans le pacte.

Au cours de la préparation du document, les parties ont partagé les revenus provenant de l'exploitation de plus grands champs de lever de soleil et positif dans le texte du pacte international la nécessité d'élaborer des règles relatives à l'exploitation des champs de la région appartenant aux deux Pays. II a été établi que les règles convenues dans le traité ou dans ses annexes ne peuvent être partiellement inaperçues par aucune des parties concernées. II a également été réglementé que des plans d'exploration de la richesse marine doivent être créés avant le début des 
procédures, ainsi que des convenus selon lequel les compagnies pétrolières devraient acheter des produits et des services. de préférence du peuple timorais.

Le pacte frontalier définitif du Timor-Leste n'est pas seulement un accord commun entre les gouvernements des deux pays. Ce pacte timorais et australien couvre également les obligations relatives à l'initiative privée. Des droits ont été attribués aux entreprises qui effectueront des activités d'exploration pétrolière et gazière dans la région des champs du Grand Lever de soleil. Ils devraient contribuer au développement du Timor-Leste.

De nombreux obstacles ont dû être levés pour que le Timor-Leste puisse se développer largement, bien qu'il ait gagné le droit d'explorer son territoire maritime. Le pétrole et le gaz sont des ressources non renouvelables, c'est-à-dire qu'ils sont des sources de revenus dont la résiliation est estimée. Mais ils sont des sources de revenus immédiats et rentables. Le développement de l'éducation, de l'infrastructure, de l'agriculture, du tourisme et de la prestation de services est essentiel à l'établissement d'une base de revenu solide et renouvelable à moyen et à long terme. Le pays est confronté à plusieurs obstacles tels que le peu d'infrastructures et le faible niveau d'éducation, mais il a connu une forte croissance depuis 2002.

Dans un monde capitaliste, il est indéniable que les ressources financières sont des facteurs déterminants pour l'évolution générale d'une nation. L'augmentation des recettes provenant de l'exploration pétrolière et gazière augmentera les recettes financières, ce qui contribuera à l'évolution de l'éducation et aux investissements dans l'infrastructure des ports et aéroports du Timor-Leste. Si, avant le Traité sur les frontières définitives, l'éducation, les infrastructures, l'agriculture et la pêche industrielle étaient des obstacles au développement du timorais, on s'attend à ce que, après le pacte définitif avec l'Australie, cette réalité soit modifiée.

\section{RÉFÉRENCES}

BRASIL. Decreto no 99.165, de 12 de março de 1990. Convenção das Nações Unidas sobre o Direito do Mar. Brasília: Planalto, 1990. Disponível em: 
https://www2.camara.leg.br/legin/fed/decret/1990/decreto-99165-12-marco-1990328535-publicacaooriginal-1-pe.html. Acesso em 15 abr.19.

DEL'OMO, Florisbal de Souza. Curso de direito internacional público. Rio de Janeiro: Forense, 2006.

GIBERTONI, Carla Adriana Comitre. Teoria e prática do direito marítimo. Rio de Janeiro: Renovar, 1998.

GOMES, Danaciano. Timor Leste: A economia do mar: um contributo para desenvolvimento sustentável. Aveiro: Mare Liberum, 2016.

GUSMÃO, Kay Raia Xanana. Breve história do mar do Timor. In: GOVERNO DO ESTADO DO TIMOR LESTE. Novas Fronteiras: conciliação histórica das fronteiras marítimas no mar do timor. Dili: Gabinete das Fronteiras marítimas, 2018.

MARCONI, Maria de Andrade, Lakatos, Eva, Maria. Fundamentos da metodologia cientifica. São Paulo: Atlas. 2003.

MARITIME BOUNDARY OFFICE. New frontiers: Timor-Leste's historic conciliation on maritime boundaries in the timor sea. [S. I.], 2015. Disponível em: http://www.gfm.tl/wp-content/uploads/2015/11/Website-map-of-Timor-Sea.pdf. Acesso em: 04 maio 2019.

MATTOS, Adherbal. Meira. O novo direito do mar. Rio de Janeiro: Renovar. 1996.

PEREIRA, Eliana Sofia da Silva. Contributo crítico para a compreensão do regime do Mar de Timor à luz do Direito Internacional. 2013. 87 f. Dissertação. (Mestrado em Ciências Jurídicas Internacionais) Faculdade de direito- Universidade Nova de Lisboa, 2013. p.14. Disponível em: https://run.unl.pt/bitstream/10362/17481/1/Pereira_2013.pdf. Acesso em: 01 abr. 2019.

REPÚBLICA DEMOCRÁTICA DE TIMOR-LESTE. Ministério das finanças. Orçamento geral do Estado 2018. Díli: Gabinete Ministerial, 2018. p. 7. Disponível 
em: https://www.mof.gov.tl/wp-content/uploads/2018/08/BB5_Port.pdf. Acesso em: 15 maio 2019.

REPUBLICA DEMOCRÁTICA DO TIMOR LESTE. História do Timor Leste. Governo do Timor Leste, Dili, [s. d.]. Disponível em: http://timor-leste.gov.tl/?p=29\&lang=pt. acesso em: 05 maio 2019.

REZEK, José Francisco. Direito internacional público: curso elementar. 10. ed. rev. e atual. São Paulo: Saraiva, 2005.

RIBEIRO, Marilda, Rosado de Sá. Direito do petróleo. 3. ed. rev. atual. e ampl. Rio de Janeiro: Renovar, 2018.

TIMOR LESTE; AUSTRÁLIA. Tratado sobre fronteiras marítimas entre Timor Leste e Austrália que estabelece as respectivas fronteiras do mar do Timor Leste. Nova York: [s.n.], 2018. p. 1. Disponível em: http://www.gfm.tl/wpcontent/uploads/2018/03/Port-Timos-Sea-Maritime-Boundary-

Treaty_Portuguese.pdf. Acesso em:04 maio 2018.

TIMOR SEA JUSTICE. All of the contested oil \& gas fields are on EastTimor's side of the median line ie closer to \#Timor than Australia. Dili, 01 mar. 2016. Tiwitte: @timor sea justice. Disponível em: https://twitter.com/TimorSeaJustice/status/704895304701763584. Acesso em: 25 maio. 2019.

ZANELLA, T. V. Curso de direito do mar. Curitiba: Juruá, 2013.

\section{APÊNDICE - REFERENCIAS DE NOTA DE RODAPÉ}

3. GOMES, Danaciano. Timor-Leste: A economia do mar: um contributo para desenvolvimento sustentável. Aveiro: Mare Liberum, 2016. 
4. GUSMÃO, Kay Raia Xanana. Breve história do mar do Timor. In: GOVERNO DO ESTADO DO TIMOR LESTE. Novas Fronteiras: conciliação histórica das fronteiras marítimas no mar do timor. Dili: Gabinete das Fronteiras marítimas, 2018. p. 6.

5. RIBEIRO, Marilda, Rosado de Sá. Direito do Petróleo. 3. ed. rev. atual. e ampl. Rio de Janeiro: Renovar, 2018.

6. RIBEIRO, Marilda, Rosado de Sá. Direito do Petróleo. 3. ed. rev. atual e ampl. Rio de Janeiro Renovar, 2018.

7. GOMES, Danaciano. Timor Leste: a economia do mar: um contributo para desenvolvimento sustentável. Aveiro: Mare Liberum, 2016.

8. MARCONI, Maria de Andrade, Lakatos, Eva, Maria. Fundamentos da metodologia cientifica. São Paulo: Atlas. 2003.

9. GUSMÃO, Kay Raia Xanana. Breve história do mar do Timor. In: GOVERNO DO ESTADO DO TIMOR LESTE. Novas Fronteiras: conciliação histórica das fronteiras marítimas no mar do timor. Dili: Gabinete das Fronteiras marítimas, 2018. p. 7-8.

10. MARITIME BOUNDARY OFFICE. New frontiers: Timor-Leste's historic conciliation on maritime boundaries in the timor sea. [S. I.], 2015. Disponível em: http://www.gfm.tl/wp-content/uploads/2015/11/Website-map-of-Timor-Sea.pdf. Acesso em: 04 maio 2019.

11. GOMES, Danaciano. Timor Leste: A economia do mar: um contributo para desenvolvimento sustentável. Aveiro: Mare Liberum, 2016. p. 35-36.

12. GOMES, Danaciano. Timor Leste: A economia do mar: um contributo para desenvolvimento sustentável. Aveiro: Mare Liberum, 2016. p. 37 e 38

13. PEREIRA, Eliana Sofia da Silva. Contributo crítico para a compreensão do regime do Mar de Timor à luz do Direito Internacional. 2013. 87 f. Dissertação. (Mestrado em Ciências Jurídicas Internacionais) Faculdade de direito- Universidade Nova de Lisboa, 2013. p.14. Disponível em: 
https://run.unl.pt/bitstream/10362/17481/1/Pereira_2013.pdf. Acesso em: 01 abr. 2019.

14. PEREIRA, Eliana Sofia da Silva. Contributo crítico para a compreensão do regime do Mar de Timor à luz do Direito Internacional. 2013. 87 f. Dissertação. (Mestrado em Ciências Jurídicas Internacionais) Faculdade de direito- Universidade Nova de Lisboa, 2013. p.14. Disponível em: https://run.unl.pt/bitstream/10362/17481/1/Pereira_2013.pdf. Acesso em: 01 abr. 2019.

15. TIMOR LESTE; AUSTRÁLIA. Tratado sobre Fronteiras Marítimas entre Timor Leste e Austrália que estabelece as respectivas fronteiras do mar do Timor Leste. Nova York: [s.n.], 2018. p. 1. Disponível em: http://www.gfm.tl/wpcontent/uploads/2018/03/Port-Timos-Sea-Maritime-BoundaryTreaty_Portuguese.pdf. Acesso em:04 maio 2018.

16. PEREIRA, Eliana Sofia da Silva. Contributo crítico para a compreensão do regime do Mar de Timor à luz do Direito Internacional. 2013. 87 f. Dissertação. (Mestrado em Ciências Jurídicas Internacionais) Faculdade de direito- Universidade Nova de Lisboa, 2013. p.14. Disponível em: https://run.unl.pt/bitstream/10362/17481/1/Pereira_2013.pdf. Acesso em: 01 abr. 2019.

17. MATTOS, Adherbal. Meira. O novo Direito do Mar. Rio de Janeiro: Renovar. 1996. p. 04

18. RIBEIRO, Marilda, Rosado de Sá. Direito do Petróleo. 3. ed. rev. atual. e ampl. Rio de Janeiro: Renovar, 2018.

19. Zona Contígua é Faixa entre o mar territorial e o alto-mar, fixada entre 12 e 24 milhas, na qual o Estado exerce sua jurisdição sobre atividades marítimas e sobre diversos interesses nacionais. 
20. Plataforma continental é definida como uma faixa de terra submersa, em toda a extensão do litoral do continente. Geralmente, a plataforma possui uma extensão de 70 a 90 km, e profundidade de 200 metros, até atingir as bacias oceânicas.

21. Nota explicativa: Águas adjacentes são aquelas que banham as margens do território de uma nação.

22. ZANELLA, T. V. Curso de Direito do Mar. Curitiba: Juruá, 2013.

23. ZANELLA, T. V. Curso de Direito do Mar. Curitiba: Juruá, 2013.

24. RIBEIRO, Marilda, Rosado de Sá. Direito do Petróleo. 3. ed. rev. atual. e ampl. Rio de Janeiro: Renovar, 2018.

25. REZEK, José Francisco. Direito Internacional Público: curso elementar. 10. ed. rev. e atual. São Paulo: Saraiva, 2005. p.307

26. BRASIL. Decreto no 99.165, de 12 de março de 1990. Convenção das Nações Unidas sobre o Direito do Mar. Brasília: Planalto, 1990. Disponível em: https://www2.camara.leg.br/legin/fed/decret/1990/decreto-99165-12-marco-1990328535-publicacaooriginal-1-pe.html. Acesso em 15 abr.19.

27. GIBERTONI, Carla Adriana Comitre. Teoria e prática do direito marítimo. Rio de Janeiro: Renovar, 1998. p.33.

28. GOMES, Danaciano. Timor Leste: A economia do mar: um contributo para desenvolvimento sustentável. Aveiro: Mare Liberum, 2016. p.51

29. PEREIRA, Eliana Sofia da Silva. Contributo crítico para a compreensão do regime do Mar de Timor à luz do Direito Internacional. 2013. 87 f. Dissertação. (Mestrado em Ciências Jurídicas Internacionais) Faculdade de direito- Universidade Nova de Lisboa, 2013. p.56. Disponível em: https://run.unl.pt/bitstream/10362/17481/1/Pereira_2013.pdf. Acesso em: 01 abr. 2019. 
30. GOMES, Danaciano. Timor Leste: A economia do mar: um contributo para desenvolvimento sustentável. Aveiro: Mare Liberum, 2016. p.110 e 111

31. RIBEIRO, Marilda, Rosado de Sá. Direito do Petróleo. 3. ed. rev. atual. e ampl. Rio de Janeiro: Renovar, 2018. P. 74 à 80

32. GUSMÃO, Kay Raia Xanana. Breve história do mar do Timor. In: GOVERNO DO ESTADO DO TIMOR LESTE. Novas Fronteiras: conciliação histórica das fronteiras marítimas no mar do timor. Dili: Gabinete das Fronteiras marítimas, 2018.

33. DEL'OMO, Florisbal de Souza. Curso de Direito Internacional Público. Rio de Janeiro: Forense, 2006. p. 292.

34. TIMOR SEA JUSTICE. All of the contested oil \& gas fields are on EastTimor's side of the median line ie closer to \#Timor than Australia. Dili, 01 mar. 2016. Tiwitte: @timor sea justice. Disponível em: https://twitter.com/TimorSeaJustice/status/704895304701763584. Acesso em: 25 maio. 2019.

35. GUSMÃO, Kay Raia Xanana. Breve história do mar do Timor. In: GOVERNO DO ESTADO DO TIMOR LESTE. Novas Fronteiras: conciliação histórica das fronteiras marítimas no mar do timor. Dili: Gabinete das Fronteiras marítimas, 2018. p. 34 e 35

36. DEL'OMO, Florisbal de Souza. Curso de Direito Internacional Público. Rio de Janeiro: Forense, 2006. p. 292

37. DEL'OMO, Florisbal de Souza. Curso de Direito Internacional Público. Rio de Janeiro: Forense, 2006. p. 292

38. REPUBLICA DEMOCRÁTICA DO TIMOR LESTE. História do Timor Leste.

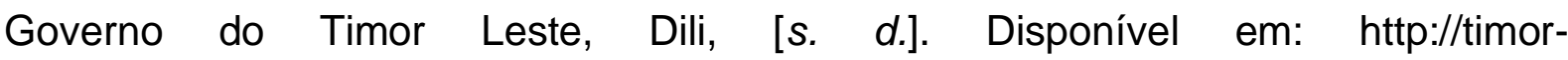
leste.gov.tl/?p=29\&lang=pt. acesso em: 05 maio 2019.

39. TIMOR LESTE; AUSTRÁLIA. Tratado sobre Fronteiras Marítimas entre Timor Leste e Austrália que estabelece as respectivas fronteiras do mar do Timor 
Leste. Nova York: [s.n.], 2018. p. 1. Disponível em: http://www.gfm.tl/wpcontent/uploads/2018/03/Port-Timos-Sea-Maritime-BoundaryTreaty_Portuguese.pdf. Acesso em:05 maio 2018.

40. PEREIRA, Eliana Sofia da Silva. Contributo crítico para a compreensão do regime do Mar de Timor à luz do Direito Internacional. 2013. 87 f. Dissertação. (Mestrado em Ciências Jurídicas Internacionais) Faculdade de direito- Universidade Nova de Lisboa, 2013. p.14-15. Disponível em: https://run.unl.pt/bitstream/10362/17481/1/Pereira_2013.pdf. Acesso em: 01 abr. 2019

41. TIMOR LESTE; AUSTRÁLIA. Tratado sobre Fronteiras Marítimas entre Timor Leste e Austrália que estabelece as respectivas fronteiras do mar do Timor Leste. Nova York: [s.n.], 2018. p. 1. Disponível em: http://www.gfm.tl/wpcontent/uploads/2018/03/Port-Timos-Sea-Maritime-BoundaryTreaty_Portuguese.pdf. Acesso em:05 maio 2018.

42. TIMOR LESTE; AUSTRÁLIA. Tratado sobre Fronteiras Marítimas entre Timor Leste e Austrália que estabelece as respectivas fronteiras do mar do Timor Leste. Nova York: [s.n.], 2018. p. 1. Disponível em: http://www.gfm.tl/wpcontent/uploads/2018/03/Port-Timos-Sea-Maritime-Boundary-

Treaty_Portuguese.pdf. Acesso em:05 maio 2018.

43. GIBERTONI, Carla Adriana Comitre. Teoria e prática do direito marítimo. Rio de Janeiro: Renovar, 1998. p. 33.

44. TIMOR LESTE; AUSTRÁLIA. Tratado sobre Fronteiras Marítimas entre Timor Leste e Austrália que estabelece as respectivas fronteiras do mar do Timor Leste. Nova York: [s.n.], 2018. p. 1. Disponível em: http://www.gfm.tl/wpcontent/uploads/2018/03/Port-Timos-Sea-Maritime-Boundary-

Treaty_Portuguese.pdf. Acesso em:05 maio 2018.

45. TIMOR LESTE; AUSTRÁLIA. Tratado sobre Fronteiras Marítimas entre Timor Leste e Austrália que estabelece as respectivas fronteiras do mar do Timor Leste. Nova York: [s.n.], 2018. p. 1. Disponível em: http://www.gfm.tl/wp- 
content/uploads/2018/03/Port-Timos-Sea-Maritime-Boundary-

Treaty_Portuguese.pdf. Acesso em:05 maio 2018.

46. TIMOR LESTE; AUSTRÁLIA. Tratado sobre Fronteiras Marítimas entre Timor Leste e Austrália que estabelece as respectivas fronteiras do mar do Timor Leste. Nova York: [s.n.], 2018. p. 1. Disponível em: http://www.gfm.tl/wpcontent/uploads/2018/03/Port-Timos-Sea-Maritime-BoundaryTreaty_Portuguese.pdf. Acesso em:05 maio 2018.

47. TIMOR LESTE; AUSTRÁLIA. Tratado sobre Fronteiras Marítimas entre Timor Leste e Austrália que estabelece as respectivas fronteiras do mar do Timor Leste. Nova York: [s.n.], 2018. p. 1. Disponível em: http://www.gfm.tl/wpcontent/uploads/2018/03/Port-Timos-Sea-Maritime-Boundary-

Treaty_Portuguese.pdf. Acesso em:05 maio 2018.

48. GOMES, Danaciano. Timor Leste: A economia do mar: um contributo para desenvolvimento sustentável. Aveiro: Mare Liberum, 2016. p. 59.

49. REPUBLICA DEMOCRÁtICA DO TIMOR LESTE. História do Timor Leste.

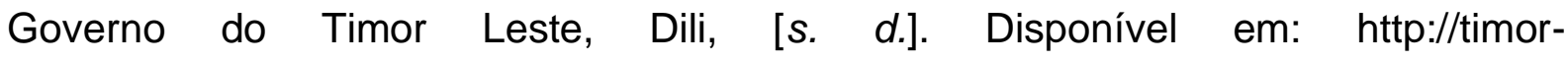
leste.gov.tl/?p=29\&lang=pt. Acesso em: 05 maio 2019.

50. O PRIMEIRO dia no Timor Leste. In: PARCEIROS pela paz. Dili, 06 jul. 2011. Disponível em: https://parceirospelapaz.wordpress.com/category/timor-leste/. Acesso em: 25 maio 2019.

51. REPÚBLICA DEMOCRÁtICA DE TIMOR-LESTE. Ministério das finanças. Orçamento geral do Estado 2018. Díli: Gabinete Ministerial, 2018. p. 7. Disponível em: https://www.mof.gov.tl/wp-content/uploads/2018/08/BB5_Port.pdf. Acesso em: 15 maio 2019.

52. TIMOR LESTE; AUSTRÁLIA. Tratado sobre Fronteiras Marítimas entre Timor Leste e Austrália que estabelece as respectivas fronteiras do mar do Timor Leste. Nova York: [s.n.], 2018. p. 1. Disponível em: http://www.gfm.tl/wp- 
content/uploads/2018/03/Port-Timos-Sea-Maritime-Boundary-

Treaty_Portuguese.pdf. Acesso em: 19 maio 2018.

53. GOMES, Danaciano. Timor Leste: A economia do mar: um contributo para desenvolvimento sustentável. Aveiro: Mare Liberum, 2016.

54. REPUBLICA DEMOCRÁtICA DO TIMOR LESTE. História do Timor Leste. Governo do Timor Leste, Dili, $\left[\begin{array}{ll}s . & d .\end{array}\right]$. Disponível em: http://timorleste.gov.t//p=29\&lang=pt. acesso em: 05 maio 2019

55. TIMOR LESTE; AUSTRÁLIA. Tratado sobre Fronteiras Marítimas entre Timor Leste e Austrália que estabelece as respectivas fronteiras do mar do Timor Leste. Nova York: [s.n.], 2018. p. 1. Disponível em: http://www.gfm.tl/wpcontent/uploads/2018/03/Port-Timos-Sea-Maritime-Boundary-

Treaty_Portuguese.pdf. Acesso em: 19 maio 2018.Acessado: 05/05/19

56. TIMOR LESTE; AUSTRÁLIA. Tratado sobre Fronteiras Marítimas entre Timor Leste e Austrália que estabelece as respectivas fronteiras do mar do Timor Leste. Nova York: [s.n.], 2018. p. 1. Disponível em: http://www.gfm.tl/wpcontent/uploads/2018/03/Port-Timos-Sea-Maritime-Boundary-

Treaty_Portuguese.pdf. Acesso em:05 maio 2018.

57. TIMOR LESTE; AUSTRÁLIA. Tratado sobre Fronteiras Marítimas entre Timor Leste e Austrália que estabelece as respectivas fronteiras do mar do Timor Leste. Nova York: [s.n.], 2018. p. 1. Disponível em: http://www.gfm.tl/wpcontent/uploads/2018/03/Port-Timos-Sea-Maritime-Boundary-

Treaty_Portuguese.pdf. Acesso em:05 maio 2018.

58. GOMES, Danaciano. Timor Leste: A economia do mar: um contributo para desenvolvimento sustentável. Aveiro: Mare Liberum, 2016

59. GOMES, Danaciano. Timor Leste: A economia do mar: um contributo para desenvolvimento sustentável. Aveiro: Mare Liberum, 2016. p.114-115. 
60. GOMES, Danaciano. Timor Leste: A economia do mar: um contributo para desenvolvimento sustentável. Aveiro: Mare Liberum, 2016.

61. REPÚBLICA DEMOCRÁTICA DE TIMOR-LESTE. Ministério das finanças. Orçamento geral do Estado 2018. Díli: Gabinete Ministerial, 2018. Disponível em: https://www.mof.gov.tl/wp-content/uploads/2018/08/BB5_Port.pdf. Acesso em: 15 maio 2019.

62. GOMES, Danaciano. Timor Leste: A economia do mar: um contributo para desenvolvimento sustentável. Aveiro: Mare Liberum, 2016.

Soumis : août 2019.

Approuvé : décembre 2019. 